\title{
Riesgo de diabetes de una comunidad rural en México: un estudio observacional
}

\author{
Diabetes Risk in a Rural Community in Mexico: an Observational Study \\ O risco do diabetes em uma comunidade rural no México: um estudo \\ observacional
}

Como citar este artículo:

Avila Sansores Grever María, Vega Argote Ma. Gloria, Ruvalcaba Palacios Gerardo, Barreto Arias Ma. Eugenia, Gomez Aguilar Patricia Isolina, Yam Sosa Antonio Vicente. Riesgo de diabetes de una comunidad rural en México: un estudio observacional. Revista Cuidarte. 2020; 11(3): e797. http://dx.doi.org/10.15649/cuidarte.797

Revista Cuidarte

Rev Cuid. 2020; 11(3): e797

doi) http://dx.doi.org/10.15649/cuidarte.797

E-ISSN: 2346-3414

(1) Grever María Avila Sansores ${ }^{1}$

(1) Ma. Gloria Vega Argote 2

(1) Gerardo Ruvalcaba Palacios ${ }^{3}$

(1) Ma. Eugenia Barreto Arias ${ }^{4}$

(1) Patricia Isolina del Socorro

Gómez Aguilar ${ }^{5}$

(1) Antonio Vicente Yam Sosa ${ }^{6}$

1 Departamento de Enfermería de la Universidad de Guanajuato, Campus Irapuato Salamanca, División Ciencias de la Vida; Guanajuato, México,E-mail: greavila@ugto.mx autor de Correspondencia.

2 Departamento de Enfermería de la Universidad de Guanajuato, Campus Irapuato Salamanca, División Ciencias de la Vida; Guanajuato, México, E-mail: gloria-vega-argote@hotmail.com

3 Departamento de Enfermería de la Universidad de Guanajuato, Campus Irapuato Salamanca, División Ciencias de la Vida; Guanajuato, México, E-mail: drruvalcaba@gmail.com

4 Departamento de Enfermería de la Universidad de Guanajuato, Campus Irapuato Salamanca, División Ciencias de la Vida; Guanajuato, México, E-mail: mbarretoarias@yahoo.com.mx

5 Facultad de Enfermería de la Universidad Autónoma de Yucatán; Yucatán México, E-mail: patricia.gomez@uady.mx

6 Facultad de Enfermería de la Universidad Autónoma de Yucatán; Yucatán México, E-mail: antonio.yam@uady.mx

\section{Resumen}

Introducción. La rápida transición del medio rural a la adopción de hábitos alimenticios de zonas urbanas, el sedentarismo, las dificultades de acceso a la salud, ponen a la población en mayor riesgo de desarrollo de diabetes, sin embargo, son pocos los estudios que se enfocan en este fenómeno a nivel rural. Conocer el riesgo permite identificar las áreas problemáticas y revertir la situación a través de modelos de atención para esta población. El objetivo de este estudio fue describir los factores de riesgo presentes en una comunidad rural de Guanajuato. Material y Métodos: Estudio descriptivo, observacional, transversal; muestra no probabilística de 164 personas sin diabetes, se calculó con Epidat 3.1, intervalo de confianza al 95\%, precisión del 3\%; se aplicó el Cuestionario de Factores de Riesgo para la Diabetes Mellitus. Para la colecta se hizo un recorrido de casa por casa hasta completar la muestra, para el análisis estadístico se utilizó la chi cuadrada y cálculo de odds ratio. Resultados: El 71\%(117) de la población fueron mujeres; el promedio para la edad fue de 43 años; el 85\% de los sujetos presentaron un alto riesgo de padecer diabetes; los tres principales factores de riesgo fueron: $57.9 \%$ (95) con sobrepeso y obesidad, el $77.4 \%(127)$ de la muestra con cintura superior al parámetro recomendado, 75.6\% (124) de la muestra presentó antecedentes heredofamiliares; en cuestión de género las mujeres presentaron 3 veces más riesgo de diabetes $(p=<.05)$. Conclusión: Los entornos rurales muy contrario a lo que se observaba, tienen alto riesgo de desarrollo de diabetes, probablemente va de la mano con los cambios de hábitos de las comunidades, pues la industrialización las ha invadido, con todo lo que conlleva, como lo señala Moreno L.

Palabras clave: Factores de Riesgo; Diabetes Mellitus; Población Rural; Peso Corporal

Recibido: mayo 8 de 2019

Aceptado: mayo 7 de 2020

Publicado: septiembre 1 de 2020 $\square *$ Correspondencia

Grever María Avila Sansores

E-mail: greavila@ugto.mx 


\section{Diabetes Risk in a Rural Community in Mexico: an Observational Study}

\section{Abstract}

Introduction: The rapid transition from rural to urban eating habits, sedentary lifestyles and poor access to healthcare put people at higher risk of getting diabetes. However, only a few studies focus on this phenomenon at the rural level. Understanding risks allow identifying problem areas and reversing the situation by using healthcare models in this population. This study is aimed to describe risk factors found in a rural community in Guanajuato. Material and Methods: A descriptive observational cross-sectional study was conducted with a non-probabilistic sample of 164 individuals without diabetes, which was calculated using Epidat 3.1, 95\% Confidence Interval and 3\% accuracy. Type 2 diabetes risk assessment questionnaire was implemented. Results: $71 \%$ (117) of the population were women at an average age of $43 ; 85 \%$ of individuals were at a high risk for diabetes. The three main risk factors were $57.9 \%$ (95) were overweight and obese, $77.4 \%$ (127) had their waist circumference above suggested parameters and $75.6 \%$ (124) had a family history. As for gender, women were three times more likely to develop diabetes $(p=<.05)$. Conclution: Rural environments, unlike previous observations, are at high risk of developing diabetes, which probably goes in parallel with changes in community habits, given that industrialization has already invaded them, including all aspects that it entails, as Moreno $L$ points out.

Key words: Risk Factors; Diabetes Mellitus; Rural Population; Body Weight

\section{O risco do diabetes em uma comunidade rural no México: um estudo observacional}

\section{Resumo}

Introdução: A rápida transição dos hábitos alimentares rurais para os urbanos, os estilos de vida sedentários e as dificuldades de acesso aos serviços de saúde põem a população em maior risco de contrair diabetes. No entanto, poucos estudos se concentram neste fenômeno no âmbito rural. O conhecimento do risco nos permite identificar as áreas problemáticas e reverter a situação através de modelos de atenção a esta população. O objetivo deste estudo foi descrever os fatores de risco presentes em uma comunidade rural de Guanajuato. Material e Métodos: Estudo observacional transversal descritivo; amostra não probabilística de 164 pessoas sem diabetes, calculada com Epidat 3.1, intervalo de confiança de 95\%, precisão de 3\%; Questionário de Fatores de Risco para Diabetes Mellitus foi aplicado. Para a coleta de dados, foi feita uma pesquisa casa a casa até a conclusão da amostra; para a análise estatística, foram utilizados o chi quadrado e cálculo dos odds ratios. Resultados: $71 \%$ (117) da população eram mulheres; a média de idade foi de 43 anos; 85\% dos indivíduos apresentaram alto risco de diabetes; os três principais fatores de risco foram: $57,9 \%$ (95) com sobrepeso e obesidade, 77,4\% (127) da amostra com a cintura acima do parâmetro recomendado, 75,6\% (124) da amostra apresentou histórico familiar; em termos de gênero, as mulheres apresentaram 3 vezes o risco de diabetes $(p=<, 05)$. Conclusão: Os ambientes rurais, ao contrário do que foi observado, têm alto risco de desenvolver diabetes, 0 que provavelmente vai junto com as mudanças de hábitos em comunidades, já que a industrialização as invadiu, com tudo o que isso implica, como aponta Moreno L.

Palavras chave: Fatores de Risco; Diabetes Mellitus; População Rural; Peso Corporal. 


\section{Introducción}

La importancia de seguir en el eje de investigar sobre la Diabetes y su afectación a la población radica en la persistencia de las cifras que se mantienen al alza, principalmente en poblaciones vulnerables; la Federación Internacional de la Diabetes (FID) en su Atlas publicado en el año 2019 señala 3 de cada 4 personas con diabetes viven en países de ingresos bajos y medios. Existen 48 millones de personas afectadas con Diabetes en América del Norte y Caribe (ANC), y una proporción de $37.8 \%$ de la población permanece sin diagnosticar. En esta región, México ocupa el segundo lugar con 12.8 millones de personas afectadas. A dichas cifras se le agrega que 56 millones de personas en esta misma región que presentan intolerancia a la glucosa por lo que tienen un alto riesgo de padecer la enfermedad. También se hace referencia que 1 de cada 2 personas con diabetes aún no está diagnosticada, y 1 de cada 6 adultos presentan intolerancia a la glucosa, la mayor cifra de todas las regiones de la FID. En cuanto a población rural se estima a nivel mundial, la prevalencia de diabetes es de 7.8\%, lo equivalente a 152,6 millones ${ }^{1}$.

En México la Encuesta Nacional de Salud y nutrición realizada en el 2018 indicó una prevalencia del $10.3 \%$, cifra que está por arriba de la mundial, con un $9.3 \% \%^{1,2}$. También ocupa el primer lugar en la Organización para la Cooperación y el Desarrollo Económico (OCDE) con el mayor gasto en salud en 2017 , con un $41.5 \%$, en tanto que el promedio de los países que lo integran es de $21 \%$. Lamentablemente como se puede ver a pesar de que es mayor el gasto en salud de dicho país, no se ve reflejado en una mejor salud de su población ${ }^{3}$.

Las enfermedades no trasmisibles como la diabetes afectan de manera desproporcional al mundo, principalmente a los sectores más pobres y de nivel cultural bajo; las desigualdades socioeconómicas en el acceso a un médico son evidentes en casi todos los países de la OCDE. Excepto Dinamarca y la República Eslovaca, las personas más ricas tienen más probabilidades de ver a un médico que las personas en el quintil de ingresos más bajos. Los problemas observados en el acceso a los servicios de salud, particularmente para los menos acomodados, ocurren a pesar de que la mayoría de los países de la OCDE tienen cobertura universal o casi uni-

1 de cada 2 personas con diabetes aún no está diagnosticada, y 1 de cada 6 adultos presentan intolerancia a la glucosa, la mayor cifra de todas las regiones de la FID. En cuanto a población rural se estima a nivel mundial, la prevalencia de diabetes es de $7.8 \%$, lo equivalente a 152,6 millones. versal de servicios de salud a través de un paquete básico. Parte de la explicación es el alto costo compartido, la exclusión de algunos servicios de los paquetes de beneficios o el racionamiento implícito de los servicios, así como las limitaciones en la alfabetización en salud, las malas estrategias de comunicación y la baja calidad de la atención también son factores contribuyentes ${ }^{4}$.

México se caracteriza por ser un país con alto grado de pobreza y desigualdad, cerca de un $23 \%$ de sus habitantes están en territorio rural; y aunque ha disminuido el porcentaje en los últimos años, aún siguen al alza los índices de pobreza; en 2016 de cada diez habitantes de zonas rurales 6 eran considerados pobres, en contraste con los 4 de cada 10 en zonas urbanas. La pobreza extrema de los hogares rurales se expresa en diferentes rezagos: entre más pequeña es la localidad existe más personas analfabetas; que no asisten a la escuela; que habitan viviendas con piso de tierra, sin energía eléctrica, que no tienen acceso al agua, y que utilizan leña o carbón para cocinar ${ }^{5}$. 
Sin embargo, son muy escasos los estudios realizados en zonas rurales ${ }^{6}$, y se hace imperiosa la necesidad de realizarlos pues en ese medio se presentan grandes obstáculos para el logro de la salud, se complica las actividades de autocuidado en los enfermos y de prevención en las personas con riesgo alto de contraer enfermedades crónicas como la diabetes ${ }^{7}$.

Cuando la enfermedad empieza a manifestarse se sabe que alrededor de $30 \%$ a $50 \%$ de las personas desconocían su problema de salud, y en zonas rurales esto ocurre casi en el $100 \%$ de los $\operatorname{casos}^{8,9}$. Ante dicha panorámica, en el escenario de la diabetes, la identificación temprana de factores de riesgo para desarrollarla permitirá implementar estrategias preventivas y de esta manera disminuir las tasas de la enfermedad ${ }^{10,11}$. así como también será de importancia la detección de los casos de diabetes que aún ignoran su estado; de la mano también está la detección de estados prediabéticos para revertir la situación ${ }^{12}$.

La OMS define como factor de riesgo, a "cualquier rasgo, característica o exposición de un individuo que aumente su probabilidad de sufrir una enfermedad o lesión. Estos factores de riesgo (biológicos, ambientales, de comportamiento, socioculturales, económicos) pueden sumarse unos a otros el efecto aislado de cada uno de ellos produciéndose un fenómeno de interacción"133, en el caso de la diabetes los factores de riesgo considerados son antecedentes heredofamiliares, la edad mayor a 45 años, sedentarismo, obesidad, entre otros ${ }^{1,13}$. De igual manera es importante remarcar que aunque el desarrollo urbano es un factor que favorece la epidemia de la Diabetes, actualmente las comunidades rurales pueden correr un mayor riesgo de sufrirla de lo que se pensaba antes; se explica que se podrían estar presentando variaciones en grados de transición, en términos de estilo de vida, economía, demografía, fenómeno de migración, y la carencia de dichos datos en términos específicos por región y comunidades pueden obstaculizar potencialmente los esfuerzos para prevenir y tratar la diabetes en los entornos rurales. El cambio de dieta tradicional en México, principalmente de los entornos rurales, basada en maíz, cereales y leguminosas, por una dieta de comida rápida e industrializada y bebidas de alta densidad energética, fomentada por la industria alimentaria, son factores predisponentes importantes que se presentan, aunados al sedentarismo cada vez más frecuente en la población ${ }^{1,5,9}$.

Por tales motivos surge la necesidad de estudiar la frecuencia de factores que podrían propiciar la aparición de Diabetes en una comunidad rural de Guanajuato, pues actualmente no se le ha dado la importancia de evaluar los riesgos en las comunidades rurales de manera que permita planear estrategias de intervención más eficaces, particularmente en el estado de Guanajuato no existen estudios que retomen la problemática desde el enfoque rural por lo que se propone realizar la presente investigación. El objetivo fue describir los factores de riesgo presentes en una comunidad rural de Guanajuato.

\section{Material y métodos}

Estudio de tipo descriptivo, observacional, transversal, no experimental; la colecta de datos se realizó del 19 de junio al 21 de julio del 2017 en una comunidad rural denominada "Ex Hacienda el Copal", en el estado de Guanajuato, que cuenta con un total de 1439 personas, con 357 hogares.

Se hizo un muestreo no probabilístico, la muestra se calculó con Epidat 3.1, con un intervalo de confianza al $95 \%$ y una precisión del 3\%, de manera que se obtuvo un total de 164 sujetos para el estudio, entre los criterios de inclusión se consideró que fueran mayores de edad, con o sin 
antecedentes heredofamiliares de diabetes, sin diagnóstico previo de diabetes, que desearan participar.

El Instrumento utilizado fue el Cuestionario de Factores de Riesgo para la Diabetes Mellitus creado por la Secretaría de Salud de México en 2015; con un total de 8 ítems, el resultado de $<10$ puntos se refiere a que el sujeto se encuentra en bajo riesgo de diabetes y se le recomendó adoptar un estilo de vida saludable, en tanto una puntuación de >10 o más indica alto riesgo de tener diabetes, en este caso se les propuso solicitar glucemia plasmática en ayuno, consultar al médico y practicar estilos de vida saludable. Sobre las variables, el mismo instrumento define para IMC: normal o adecuado un valor entre 18.5 y 24.9, inadecuado cuando se presenta un valor $\geq 25$; las puntuaciones para el riesgo consideradas fueron cero si se encontró en el parámetro normal, 5 si presentó sobrepeso y 10 si presentó algún grado de obesidad. Para la variable cintura, se consideró como riesgo en mujeres si presentó $\geq 80 \mathrm{~cm}$. y para hombres $\geq$ $\underline{90} \mathrm{~cm}$., para los valores encontrados menores a éstos se consideró cero riesgo, en tanto si presentó dichos valores se consideró 10 puntos. Para ejercicio, si hace poco o nada se consideró 5 puntos, cero si acostumbra a realizarlo. Para la edad, se consideró de cero riesgo si se encontró $<45$ años, de 5 puntos si se encontró entre 45 y 64 años, de 9 puntos para edad $\geq 65$ años. Para antecedentes familiares, se consideró de riesgo con puntuación de 1, cuando los presentó. Por último, el instrumento señala 1 punto para las mujeres que han tenido bebés mayores de $4 \mathrm{~kg}$.

Para la colecta de datos se realizó visitas domiciliarias de casa por casa, a través de entrevistas, se procuró hacer un recorrido en todo el poblado y abarcar los diferentes sectores, la colecta se realizó de julio a agosto del 2018. En primera instancia se visitó al sujeto, después que firmó el consentimiento informado se pactó la cita por la mañana para realizar la entrevista y las mediciones antropométricas, a la par que se le explicó las condiciones para realizarla.

Respecto al análisis estadístico se utilizó el programa SPSS ver. 21; para los datos Categóricos se utilizaron tablas y gráficas con porcentajes descriptivos de los mismos dado la naturaleza de las variables, en tanto que para las variables numéricas se utilizaron medidas de dispersión y tendencia central (media, mediana, mínimo, máximo), para el análisis estadístico se utilizó una Chi cuadrada y cálculo de Odds ratio para identificar el riesgo de padecer diabetes.

Se respetó la dignidad y los derechos humanos de cada persona, teniendo como marco a la declaración de Helsinki, el principal propósito de esta investigación fue para mejora de los procedimientos preventivos y terapéuticos de salud, se tomó como eje los principios éticos, legales y jurídicos correspondientes ${ }^{14}$. La investigación se apegó a lo dispuesto en el reglamento de la Ley General de Salud Mexicana en materia de investigación en su título segundo a fin de asegurar el respeto a la dignidad de los sujetos y la protección de sus derechos y bienestar. Se consideró una investigación de no riesgo (Título segundo: De los Aspectos Éticos de la Investigación en Seres Humanos, Capítulo I, Artículo 17) ya que solo se colectaron datos que no ocasionaron ninguna afectación física o psicológica ${ }^{15}$.

\section{Resultados}

En relación a la descripción de variables sociodemográficas, el género que predominó fueron las mujeres con un $71 \%(117)$ del $100 \%$ (164); en relación con la edad, la media para el total de la muestra fue de 43 años con una desviación de 17.1; con respecto a la ocupación se observó principalmente dedicarse a labores domésticas con un $77.8 \%$ (108), seguido de otras ocupaciones con un $12.8 \%$ (21) entre las que cabe destacar el comercio informal. 
Con respecto a los factores de riesgo presentes en la población rural fueron: el índice de masa corporal (IMC) de la muestra se encontró entre sobrepeso y obesidad en el $57.9 \%$ (95) de las personas; el 77.4\%(127) de la muestra presentaron una cintura superior al parámetro recomendado; un 47\%(78) realiza ejercicio; de acuerdo a la edad con mayor riesgo de diabetes, es decir personas con 45 años o más, la obtuvieron el 43.9\%(72) de la población de estudio (ver tabla 1); en relación a los antecedentes heredofamiliares el 75.6\% (124) presentó antecedentes, de los cuáles el 42\%(69) principalmente tiene a los padres con diabetes (ver gráfica 1).

Tabla 1. Factores de riesgo de diabetes por género. Irapuato, Guanajuato. 2018

\begin{tabular}{|c|c|c|c|c|}
\hline Factor de Riesgo & $\begin{array}{c}\text { Total } \\
164 \\
\end{array}$ & $\begin{array}{c}\text { Hombres } \\
47 \\
\end{array}$ & $\begin{array}{c}\text { Mujeres } \\
117 \\
\end{array}$ & Valor $p$ \\
\hline IMC \%(n) & & & & 0.139 \\
\hline Adecuado & $40.8(67)$ & $51.1(24)$ & $38.5(45)$ & \\
\hline Inadecuado & $57.9(95)$ & $48.9(23)$ & $61.5(72)$ & \\
\hline Cintura \%(n) & & & & 0.001 \\
\hline Adecuado & $22.5(37)$ & $40.4(19)$ & $15.4(18)$ & \\
\hline Inadecuado & $77.4(127)$ & $59.6(28)$ & $84.6(99)$ & \\
\hline Realiza ejercicio & $47(78)$ & $55.3(26)$ & $44.3(52)$ & 0.204 \\
\hline Edad & & & & 0.510 \\
\hline$<45$ años & $54.8(90)$ & $59.6(28)$ & $53.9(62)$ & \\
\hline$\geq 45$ años & $43.9(72)$ & $40.4(19)$ & $46.1(53)$ & \\
\hline $\begin{array}{l}\text { Antecedentes de familiar } \\
\text { con diabetes }\end{array}$ & & & & 0.164 \\
\hline $\mathrm{Si}$ & $75.6(124)$ & $83(39)$ & $72.6(85)$ & \\
\hline No & $24.3(40)$ & $17(8)$ & $27.4(32)$ & \\
\hline Riesgo & & & & 0.01 \\
\hline Alto & $85.4(140)$ & $74.4(35)$ & $89.7(105)$ & \\
\hline Bajo & $14.6(24)$ & $25.5(12)$ & $10.2(12)$ & \\
\hline
\end{tabular}

Fuente: Cuestionario de Factores de Riesgo de Diabetes Mellitus. $n=164$

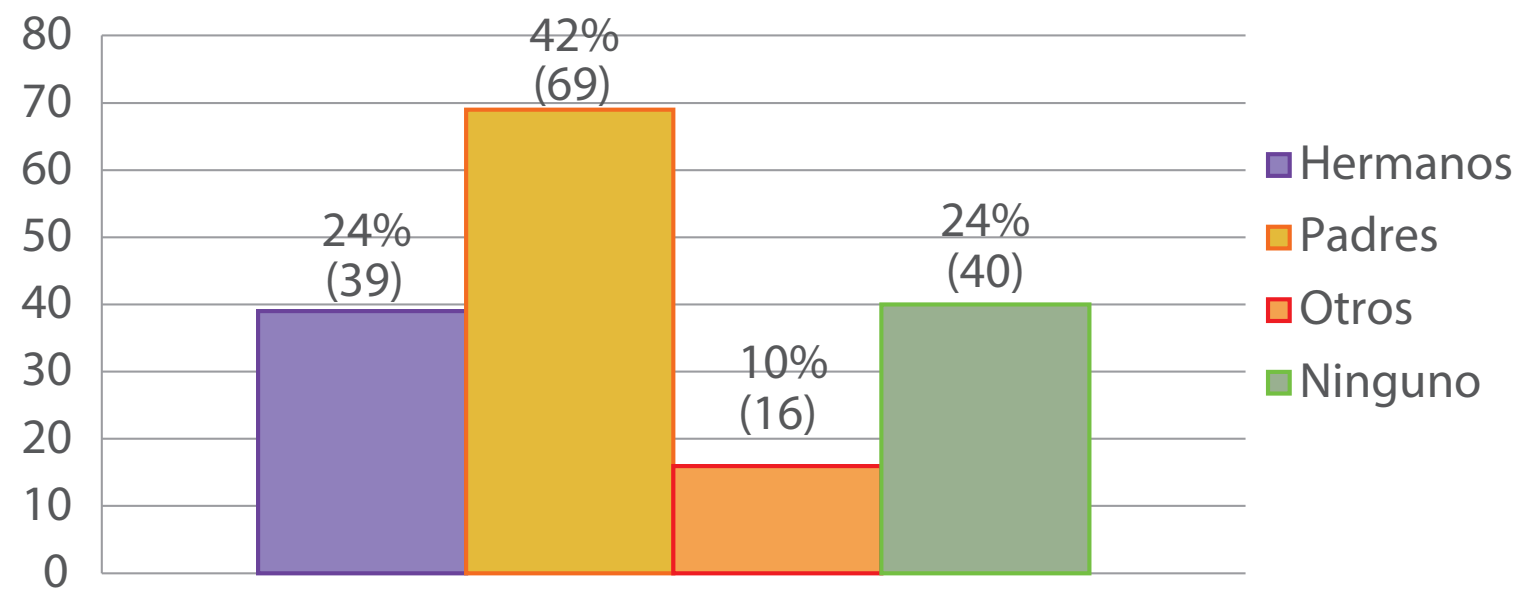

Gráfica 1. Antecedentes Heredofamiliares Irapuato, Gto 2018

Fuente: Cuestionario de Factores de Riesgo de Diabetes Mellitus. $n=164$ 
Al clasificar el riesgo de padecer Diabetes en la población en general acorde al cuestionario aplicado se encontró que el 85\% (140) se encontró en un alto riesgo de padecer la enfermedad (ver gráfica 2).

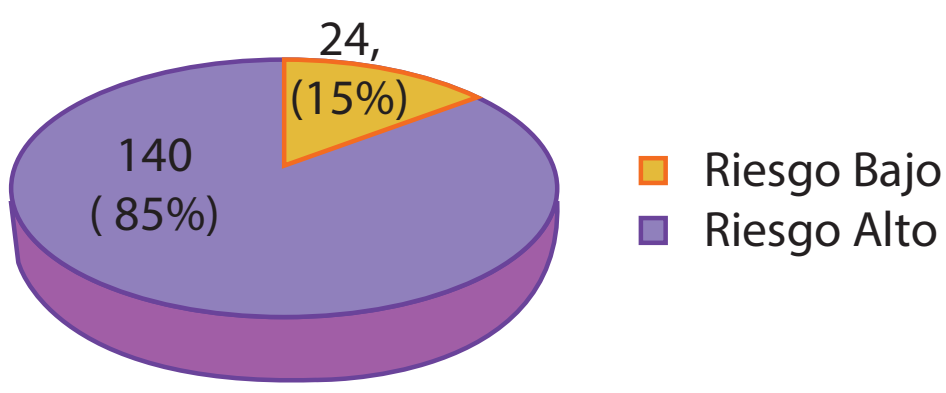

Gráfica 2. Grado de Riesgo de padecer Diabetes. Irapuato, Gto. 2018

Fuente: Cuestionario de Factores de Riesgo de Diabetes Mellitus. $n=164$

En la descripción por género, el femenino obtuvo un 75\% (105) en alto riesgo de padecer diabetes en comparación con un $25 \%(35)$ en hombres, con una $X^{2}(g l=1, N=164)=6.26, p .=01$. En relación con el cálculo de riesgo con Odds Ratio (OR) se encontró que las mujeres presentan 3 veces más riesgo de padecer diabetes que los hombres $(z=2.42 ; \mathrm{IC}: 1.23-7.28 ; \mathrm{p}=.01)$.

En relación al análisis estadístico, al comparar la frecuencia de los diferentes factores de riesgo por género como se puede ver en la tabla 1, únicamente se encontró diferencia estadísticamente significativa para la variable cintura, en donde las mujeres la presentaron más elevada en el $84.6 \%$ (99) de las mismas en comparación con 59.6\% (28) de los hombres, con una $x^{2}=12.03 ; p .=001$; para las demás variables aunque no se encontró diferencias estadísticamente significativas se observaron porcentajes importantes elevados, como por ejemplo, en comparación con los hombres, las mujeres presentaron principalmente un IMC elevado (>25) en el 61.5\% (72); en cuanto a la práctica de ejercicio el porcentaje más alto fue para las mujeres con un $55.7 \%(65)$ que refieren no realizarlo; en relación a la edad los hombres tienen ventaja pues presentaron una edad menor de 45 años en el 59.6\%(28) de ellos; en cuanto a la presencia de antecedentes heredofamiliares los hombres obtuvieron un mayor porcentaje en un $83 \%(39)$ de los mismos.

Además de lo anterior, también se observó que las mujeres con antecedentes de bebés macrosómicos (>4kg), del 100\% (117) de las mujeres un 20\% (27) señalaron haber tenido bebés con peso mayor a $4 \mathrm{~kg}$.

\section{Discusión}

El presente estudio se trabajó en una zona rural de Guanajuato y se encontró un alto riesgo de padecer Diabetes en un $85 \%$ de la población, lo cual es alarmante pues comparado con un estudio realizado en una comunidad rural de Venezuela por Brito N. y cols. en 2014 encontraron que el $21.95 \%$ de los encuestados estaban en alto riesgo de desarrollar diabetes tipo 2 , siendo las mujeres las más afectadas significativamente $(p=0,047)^{16}$. Los resultados del incremento de las enfermedades crónicas van ligadas a los efectos de la globalización, y el ambiente rural no es la excepción, pues ya se observa la invasión en éstos con alimentos industrializados en vez 
de los étnicos tradicionales, el abandono del campo para trabajar en fábricas, el sedentarismo en parte causado por las horas de traslado de una comunidad al trabajo, las largas jornadas laborales han propiciado un cambio en los estilos de vida y en consecuencia el riesgo a padecer cronicidades ${ }^{17,18}$.

De los factores de riego presentes en la población rural estudiada, el sobrepeso y obesidad lo presentó un 50\%; dicha variable va de la mano con el índice de cintura, en donde el mayor porcentaje fue para el género femenino $>80 \mathrm{~cm}$ en el $84.6 \%$ de las mujeres, sin embargo también fue elevado en hombres en un $59.6 \%$ de ellos. La presencia de dichos factores, principalmente la obesidad, se asocia a su vez a un incremento de grasa visceral de corazón, hígado, páncreas; la acumulación de grasa ectópica se asocia a presencia de resistencia a la insulina, hipertensión, dislipidemia aterogénica, inflamación endotelial, riesgo de trombosis, que termina en el desarrollo de enfermedad cardiovascular ${ }^{19}$.

Un estudio realizado por Plessis en 2018 en una población de Argentina, encontró que el 18\% (20) de un total de 113 sujetos presentaron riesgo alto de padecer diabetes en los próximos 10 años. Existió correlación positiva significativa entre la razón cintura talla $(\mathrm{RCT})$ y la razón cintura cadera (RCC) con el riesgo de padecer diabetes $(r=0,65)$. Se halló mayor frecuencia de riesgo de Diabetes en personas con sobrepeso y obesidad ${ }^{20}$. A pesar de que este estudio es mucho más bajo el riesgo detectado que el realizado en esta presente investigación, llama la atención los datos de sobrepeso y obesidad altos que son equiparables.

Cabe señalar que una de las medidas que se han implementado en México son los impuestos a las bebidas azucaradas en 2014, y uno de los efectos que se encontró fue una reducción de aproximadamente $6 \%$ en las compras de las bebidas azucaradas en 2014, en comparación con 2013, y la reducción en el grupo socioeconómico del percentil más pobre redujo la compra en $17 \%$ en diciembre de ese mismo año ${ }^{21}$. La Conferencia de Otawa plantea la necesidad de desarrollar 5 grandes estrategias en materia de promoción de la salud: formulación de políticas públicas; creación de entornos saludables; movilización consiente y empoderada de la población; desarrollo de las capacidades de las personas para enfrentar problemas y tomas decisiones apropiadas para el cuidado de su salud; y reorientar los servicios de salud hacia la comunidad y el cuidado de la salud ${ }^{22}$.

Otro estudio realizado por Llorente CY, Miguel-Soca PE, Rivas VD, y cols, encontró el riesgo de diabetes mellitus tipo 2 es mayor en pacientes con antecedentes familiares de la enfermedad, obesos, hipertensos y portadores de síndrome metabólico ${ }^{23}$. Para esta presente investigación se observó un porcentaje alto, con un $76.5 \%$ de personas con antecedentes heredofamiliares, lo cual refleja un riesgo latente del desarrollo de la enfermedad. Petermann y cols. en 2018 en un estudio realizado observó que las personas físicamente inactivas y con antecedentes familiares de la enfermedad, presentaron una mayor probabilidad de desarrollar diabetes en comparación con aquellas físicamente activas y sin antecedentes. Este mismo autor recomienda realizar $\geq 600 \mathrm{MET} / \mathrm{minutos} /$ semana de actividad física para disminuir la probabilidad de desarrollarla ${ }^{24}$. 
Para esta investigación se encontró que el riesgo es más alto de diabetes en mujeres; Chen D y cols. en un estudio realizado en 2017 en Santiago Atitlán, comunidad rural guatemalteca indígena con altos tasas de pobreza y retraso en el crecimiento, observó altas tasas de obesidad, particularmente en mujeres. Además, se encontraron altas tasas de hipertensión y dislipidemia, pero una baja tasa de diabetes mellitus. En relación a las diferencias de género, se explica que los hombres suelen realizar trabajos intensivos físicamente, mientras que las mujeres tienen más probabilidades de trabajar en casa, en los mercados o en otras ocupaciones que requieren menos actividad física, lo que puede repercutir en su salud. Alternativamente, es posible que las influencias genéticas predispongan a estas mujeres a la obesidad central ${ }^{25}$.

Dentro de las limitaciones del estudio se identifica que no existen instrumentos que sean especiales para poblaciones rurales, por lo que se aplicó uno que estaba disponible en

En relación a las diferencias de género, se explica que los hombres suelen realizar trabajos intensivos físicamente, mientras que las mujeres tienen más probabilidades de trabajar en casa, en los mercados o en otras ocupaciones que requieren menos actividad física, lo que puede repercutir en su salud. México para identificación de factores de riesgo de diabetes. No se realizaron mediciones biofisiológicas por falta de recursos económicos como por ejemplo, glucosa, colesterol triglicéridos y hemoglobina glicosilada, lo que permitiría una mejor determinación y en su caso diagnóstico del estado de salud de la población. No se aplicó un instrumento para un mejor análisis de actividad física/ejercicio.

\section{Conclusión}

Existe un alto riesgo en el que se encuentran las comunidades rurales de padecer diabetes, particularmente la estudiada, pone de manifiesto que aún falta doblegar esfuerzos es estas áreas, donde está latente la enfermedad; el profesional de enfermería debe retomar las estrategias a través de modelos de prevención, con enfoque de esta población en riesgo, adaptados culturalmente.

Por otro lado, de acuerdo a lo observado en esta presente investigación el sobrepeso y la obesidad muy contrario a lo que se pensaba hace algunos años, ya esta presente en las comunidades rurales en donde prevalecía la desnutrición y bajo peso. Poner especial atención en el IMC como factor de riesgo de enfermedades crónicas, que hasta cierto punto es modificable, las estrategias de gestión por parte de enfermería con medios gubernamentales para generar propuestas de acondicionamiento de áreas libres y seguras donde se impulsen actividades recreativas y de actividad física, pues mucho del recurso para estos espacios se queda en las grandes ciudades. Rescatar la dieta tradicional a través de campañas educativas, que no solo sean transmisoras de información, si no que involucren de manera activa a las personas, considerando su cultura, economía, disponibilidad de alimentos, nivel educativo.

Conflictos de interes: Los autores manifiestan no tener conflictos de intereses.

Financiación: Universidad de Guanajuato, Departamento de Enfermería y Obstetricia. 


\section{Referencias}

1. Federación Internacional de Diabetes. Atlas de la Diabetes de la FID. 9a ed. Bruselas: FID; 2019. https://www.diabetesatlas.org/es/resources/

2. Instituto Nacional de Salud Pública. Encuesta Nacional de Salud y Nutrición 2016. México: INS; 2016

3. Organisation for Economic Co-operation and Development. Health at a Glance 2017. Paris: OECD; 2017. http://dx.doi.org/10.1787/health_glance-2017-en

4. Organisation for Economic Co-operation and Development. Health at a Glance 2019. Paris: OECD; 2019. https://www.oecd.org/

5. Organización de las Naciones Unidas para la Alimentación y la Agricultura. México rural del Siglo XXI. México: FAO;2018.

6. González N, Valadez I, Morales A, Ruvalcaba N. Sub-diagnóstico de diabetes y prediabetes en población rural. Rev Salud Pública y Nutr. 2016;15(4):9-13. https://www.medigraphic.com/pdfs/revsalpubnut/spn-2016/spn164b.pdf

7. Villalobos A, Rojas-Martínez R, Aguilar-Salinas CA, Romero-Martínez M, Mendoza-Alvarado $\mathbf{L R}$, Flores-Luna $\mathbf{M}$ de $\mathrm{L}$, et al. Atención médica y acciones de autocuidado en personas que viven con diabetes, según nivel socioeconómico. Salud Publica Mex. 2019;61(6):876. https://doi.org/10.21149/10546

8. Santiago Mijangos AD, Lara Hernández J, Martínez Ruíz E, Jiménez Zúñiga EA, Pérez Fonseca $\mathbf{M}$, et al. Factores y nivel de riesgo para diabetes mellitus tipo 2 e hipertensión arterial en una comunidad rural. Rev. de Alta Tecnología y la Sociedad. 2017;9(4):50-6

9. Moreno-Altamirano L, García-García JJ, Soto-Estrada G, Capraro S, Limón-Cruz D. Epidemiología y determinantes sociales asociados a la obesidad y la diabetes tipo 2 en México. Rev Médica del Hosp Gen México. 2014 Jul;77(3):114-23.

https://doi.org/10.1016/j.hgmx.2014.07.002

10. Sarabia A. Betty, Can V. Ana GCJ. Identificación de Factores de Riesgo de la Diabetes Mellitus Tipo 2 en Adultos de 30 a 60 Años de edad en la Comunidad de Isla Aguada, Municipio de ciudad del Carmen, Campeche. Rev Iberoamericana para la Investigación y el Desarrollo Educativo.2016;5(10):1-15. https://dialnet.unirioja.es/servlet/articulo?codigo=5435234

11. Pérez Rodríguez A, Berenguer Gouarnaluses M. Algunos determinantes sociales y su asociación a la diabetes mellitus tipo 2. Medisan. 2015;19(10):3065-6

12. Zhao M, Lin H, Yuan Y, Wang F, Xi Y, Wen L, et al. Prevalence of pre-diabetes and its associated risk factors in rural areas of Ningbo, China. Int J Environ Res Public Health. 2016;13(8):113. https://doi.org/10.3390/ijerph13080808

13. Organización Mundial de la Salud. Temas de Salud: Factores de Riesgo. Ginebra:OMS; 2016. http://www.who.int/topics/risk_factors/es/

14. World Medical Association. Declaration of Helsinki - Ethical Principles for Medical Research Involving Human Subjects. France:World Medical Association, Inc.; c2015. https://www.wma. net/policies-post/wma-declaration-of-helsinki-ethical-principles-for-medical-research-inv

15. Secretaría de Salud. Reglamento de la Ley General de Salud en materia de Investigación para la Salud. México: Secretaría de Salud. 2014.

http://www.salud.gob.mx/unidades/cdi/nom/compi/rlgsmis.htm

16. Brito Núñez NJ, Brito Núñez JD, Ruiz-Rendón CM. Riesgo de diabetes en una comunidad rural del municipio Sotilo: Estado Monagas, Venezuela. Rev Venezolana Endocrinol Metab. 2014;12(3):167-76.

http://ve.scielo.org/scielo.php?script=sci_arttext\&pid=S1690-31102014000300004 
17. Román, S., Ojeda, C. y Panduro, A. Genética y evolución de la alimentación de la población en México. Revista de Endocrinología y Nutrición. 2013;20(1):42-51.

18. Organización de las Naciones Unidas para la Alimentación y la Agricultura. Transición de la Nutrición y Obesidad; 2018. http://www.fao.org/FOCUS/S/OBESITY/obes2.htm

19. Bryce-Moncloa, Alfonso, Alegría-Valdivia, Edmundo, \& San Martin-San Martin, Mauricio G. Obesidad y riesgo de enfermedad cardiovascular. Anales de la Facultad de Medicina. 2017;78(2):202-206. https://doi.org/10.15381/anales.v78i2.13218

20. du Plessis, María Cristina. Relación entre el riesgo de padecer diabetes mellitus e índices antropométricos en una localidad rural de Tucumán, Argentina. Anales de la Facultad de Medicina. 2018;79(3):206-212. https://doi.org/10.15381/anales.v79i3.15340

21. Instituto Nacional de Salud Pública. Resultados preliminares sobre los efectos del impuesto de un peso a bebidas azucaradas en México. México; 2018.

https://www.insp.mx/epppo/blog/preliminares-bebidas-azucaradas.html

22. Organización Panamericana de la Salud. Carta de Otawa. OPS; 1986. https://www.paho. org/hq/dmdocuments/2013/Carta-de-ottawa-para-la-apromocion-de-la-salud-1986-SP.pdf

23. Llorente CY, Miguel-Soca PE, Rivas VD, et al. Factores de riesgo asociados con la aparición de diabetes mellitus tipo 2 en personas adultas. Rev Cuba Endoc. 2016;27(2):123-133.

24. Petermanna Fanny, Díaz-Martínezb Ximena, Garrido-Méndezc Álex, Leivad Ana María, Martíneze María Adela, Salasf Carlos, et al. Asociación entre diabetes mellitus tipo 2 y actividad física en personas con antecedentes familiares de diabetes. Gac Sanit. 2018;32(3):230235. https://doi.org/10.1016/j.gaceta.2017.09.008

25. Chen D, Rivera-Andrade A, González J, Burt D, Mendoza-Montano C, Patrie J, Luna M. Prevalence of risk factors for noncommunicable diseases in an indigenous community in Santiago Atitlán, Guatemala. Rev Panam Salud Publica. 2017;41:e7.

https://doi.org/10.26633/RPSP.2017.7 